\title{
GTE-Cluster: A Temporal Search Interface for Implicit Temporal Queries http://wia.info.unicaen.fr/GTEAspNetFlat TempCluster_Server
}

\section{Download this poste \\ E}

36th European Conference on Information Retrieval (ECIR 2014) Amsterdam, Netherlands. 13-16 April 1. 2014.

\section{ABSTRACT}

Given that most of the temporal queries issued by users are implicit by nature [2], detecting its underlying temporal intent turns out to be a very interesting problem and a real need to improve the performance of search

In this context, most state-of-the-art methodologies consider any occurrence of temporal expressions in Web snippets and other Web data, as equally relevant to an implicit temporal query. This is obviously not true for most part of the query results.

\section{Haiti Earthquake Anniversary}

As of 2010 (see 1500 photos), the following major earthquakes have been recorded in Haiti. The 1st one occurred in however in 2012 Haiti will organize the Carnival...

has been a tragic date queries in order to further improve the Web search process.

For that purpose, we propose a language-independent strategy to associate top relevant years to any text query by analyzing its corresponding Web

snippets. 1. Select the most relevant dates for a given query 2. Discard all non-relevant or incorrect ones;

The ability to identify relevant dates automatically is potentially useful for several tasks, including temporal clustering [3].

In this demo, we present GTE-Cluster an online temporal search interface which consistently allows searching for topics in a temporal perspective by clustering relevant temporal Web search results.

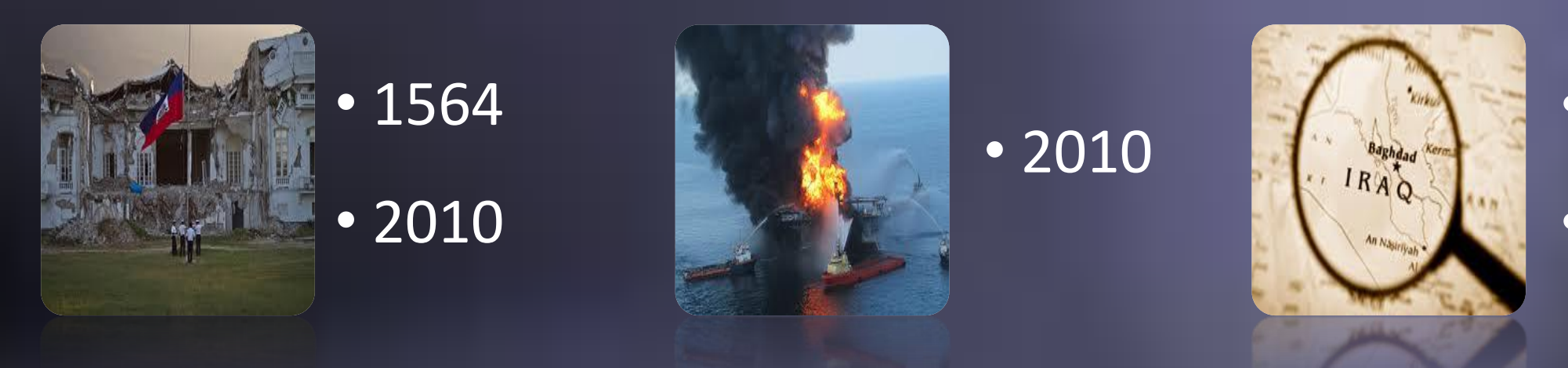

Our A pproach

ARCHITECTURE
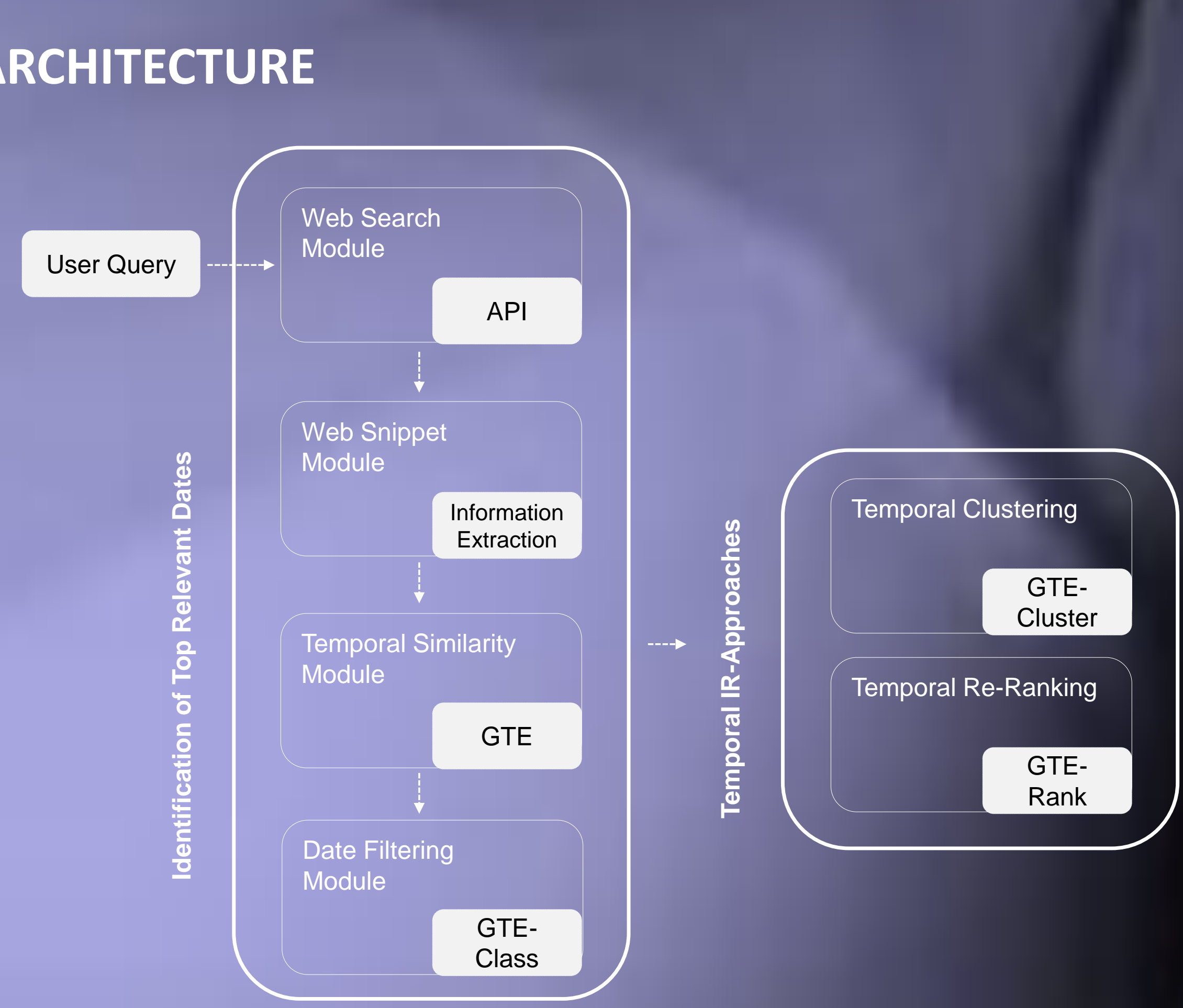

ALGORITHM

Algorithm: Determine the list of clusters for the query $a$

Input: query $q$

1: $S \leftarrow$ GetSnippetsFromSearchEngine $(q)$

For each $S_{i} \in S, \mathrm{i}=1, . ., \mathrm{n}$

Apply Text Processing

$D_{S_{i}} \leftarrow$ Select all temporal patterns in $S_{i}$

6: $W_{S} \leftarrow U_{i=1}^{n} W_{S}$

7: $D_{S} \leftarrow U_{i=1}^{n} D_{S_{1}}$

9: For each $d_{j} \in D_{s}$

10: Compute $\operatorname{GTE}\left(q, d_{j}\right)$

11: $D_{S}^{\text {Rel }} \leftarrow$ Determine the final list of $m$ relevant dates by applying GTE-Class

12: $C_{j} \leftarrow \varnothing$

13: For each $d_{j} \in D_{S}^{R e l}, \mathrm{j}=1, . ., \mathrm{m}$

14 For each $S_{i} \in S, \mathrm{i}=1, \ldots, \mathrm{n}$

if $S_{i}$ has $d_{j}$ then

16: $\quad C_{j}+=s_{i}$

TEMPORAL SIMILARITY MODULE (GTE)

$\operatorname{GTE}\left(q, d_{j}\right)=\operatorname{sim}\left(q, d_{j}\right) \in[0,1]$

\begin{tabular}{|c|c|c|}
\hline $\begin{array}{c}\text { Gaël Dias } \\
\text { HULTECH/GREYC, } \\
\text { University of Caen Basse-Normandie, } \\
\text { France } \\
\text { gael/dias@unicaen.fr } \\
\text { http://dias.users.greyc.fr }\end{array}$ & $\underset{\text { INESCTEC }}{\operatorname{LAAD}}$ & $\begin{array}{c}\text { Alípio Mário Jorge } \\
\text { LIAAD - INESC Porto, LA } \\
\text { DCC - FCUP } \\
\text { University of Porto, Portugal } \\
\text { amjorge@fc.up.pt } \\
\text { http://www.liaad.up.pt/ amjorge/ }\end{array}$ \\
\hline
\end{tabular}

Our proposal is that the relevance between a pair is better defined if, instead of just focusing on the self-similarity (between the query and the date), we calculate all the similarities existing between the date and each $\operatorname{GTE}\left(q, d_{j}\right)=F\left(\operatorname{sim}\left(w_{j}, d_{j}\right)\right), w_{j} \in W^{*}$

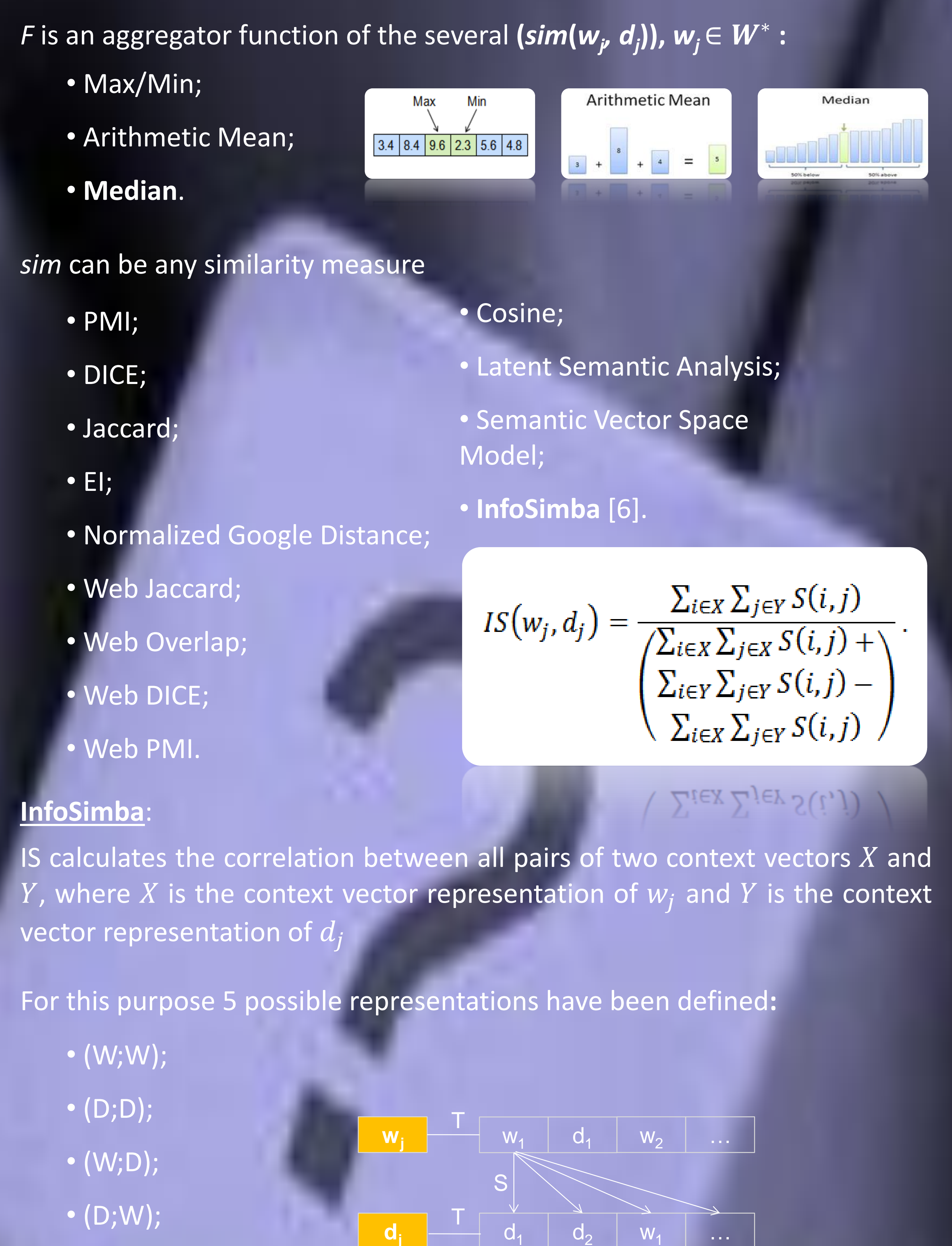

$\cdot(W D ; W D)$.

$S$ is any similarity measure, such as PMI, DICE, Jaccard, etc.

$\boldsymbol{T}$ is the minimum similarity value above which, words and candidate dates should be selected as elements of the two context vectors

For that end, a conceptual temporal correlation matrix $\mathrm{M}_{\mathrm{ct}}$, which stores the similarity between the most important words and the candidate dates is built;
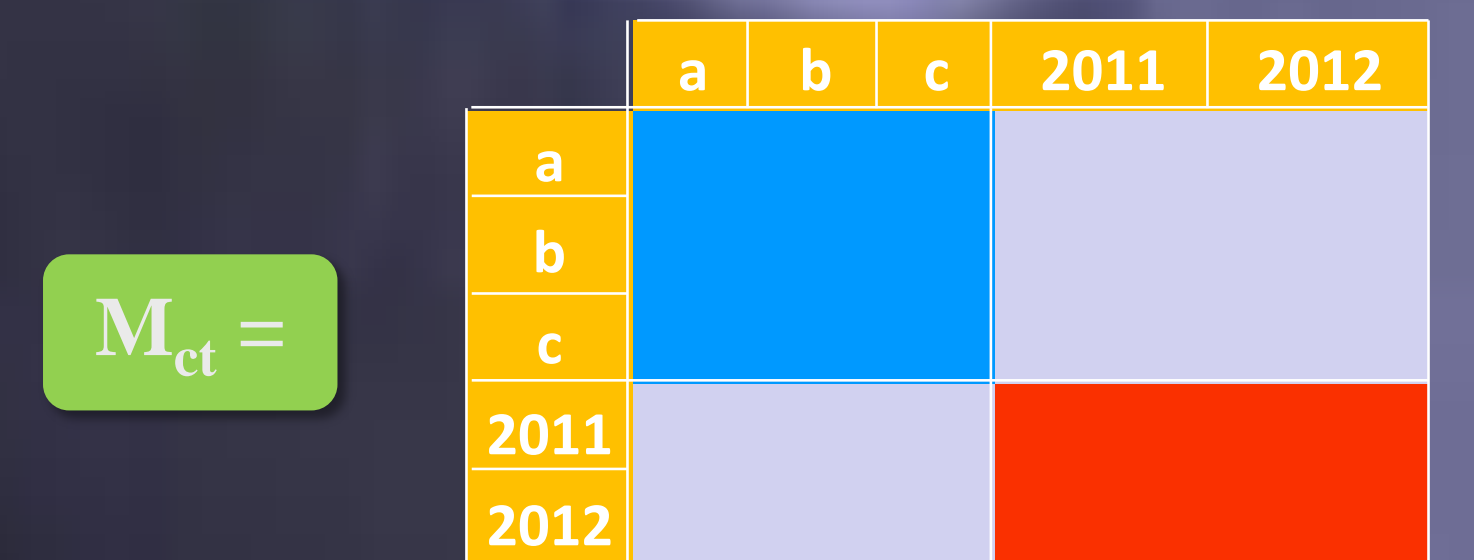

DATE FILTERING MODULE (GTE-Class):

Classical threshold-based strategy [4];

1. relevant, if $\left(\operatorname{GTE}\left(q, d_{j}\right) \geq \lambda\right)$

2. non-relevant, if $\left(\operatorname{GTE}\left(q, d_{j}\right)<\lambda\right)$

TEMPORAL CLUSTERING (GTE-Cluster):

GTE-Cluster focuses on adding top relevant temporal features to postretrieval clustering based on the following principle:

P1: Two snippets are temporally similar if they are highly related to the e set of dates.

Each web snippet $S_{i}$ contains a set of $D_{S_{i}}^{\text {Rel }}$ dates, which directly reflect the web snippet temporal purpose.

Since its text can contain several different relevant temporal features, we permit overlapping: each snippet $S_{i}$ may belong to a number of $m$ clusters

The final set of clusters consists of $m$ entities, where $m$ is the number of relevant dates in $D_{S}^{R e l}$

The set of clusters are then sorted in ascending order by date.

One such presentation of the results enables users to have a quick overview of a topic, without the need to go through an extensive list of One of the advantages of our clustering model is that instead of considering all the temporal expressions as equally relevant as in [1], we determine which ones are more relevant to the user text query.

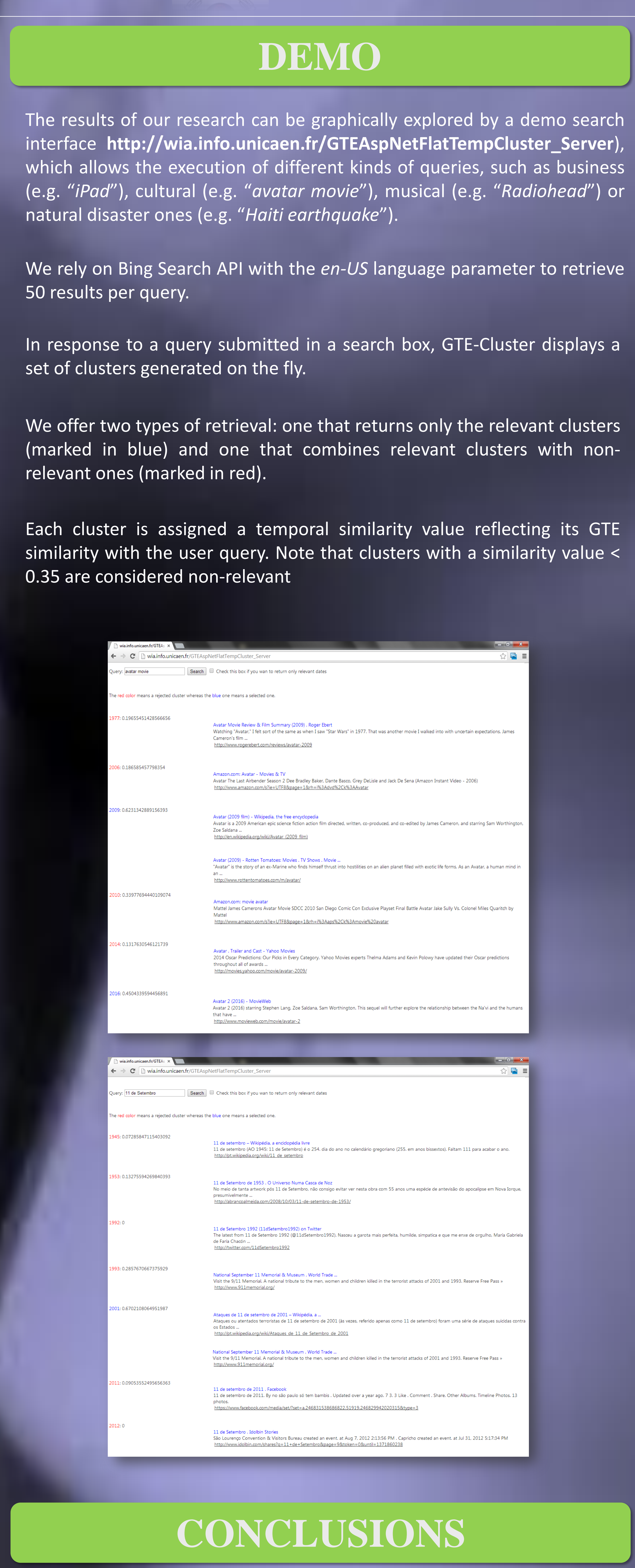

- In this paper, we presented GTE-Cluster a temporal search interface that focuses on disambiguating a text query with respect to its temporal purpose(s);

-We proposed a strategy for temporal clustering of Web search results, where snippets are clustered by year;

-We believe that the introduction of the temporal dimension will help to mitigate the limitations that users experience when their information needs include topics of a temporal nature

- This is our first version approach to flat temporal clustering of search results. While we already achieved an initial stage of flat clustering by time our proposal still lacks an approach focused on topics;

- As future research, we aim to provide an effective clustering algorithm that clusters and ranks snippets, both based on their temporal and conceptual proximities;

- A future approach should also consider a more elaborated mechanism in terms of ranking by applying an inter-cluster and an intra-cluster solution:

-Finally, we may use the similarity value associated to each cluster to offer an ordered set of temporal query suggestions.

\section{REFERENCES}

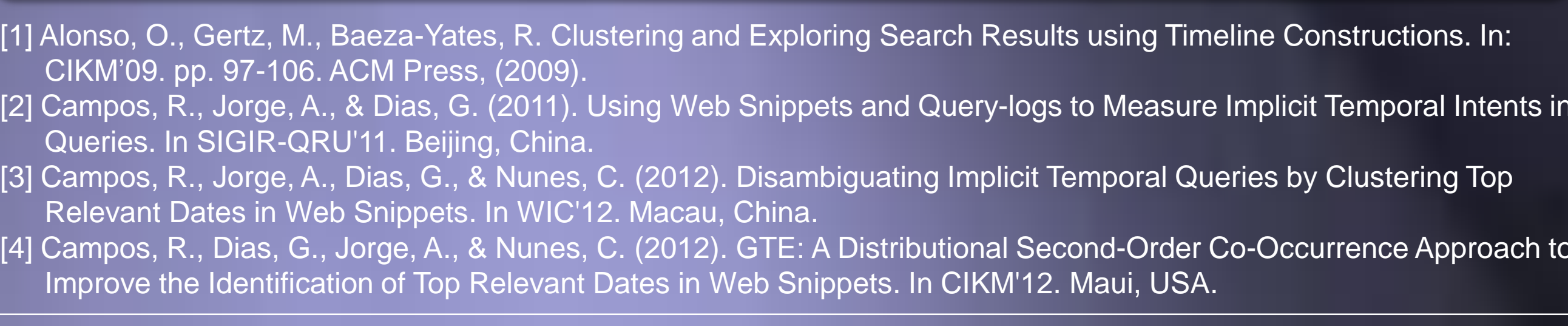

